\title{
Fatores Relacionados ao Atraso no Diagnóstico de Câncer de Boca e Orofaringe em Juiz de Fora/MG
}

\author{
Associated Factors for the Delay in the Diagnosis of Oral and Oropharynx Cancer \\ in Juiz de Fora/MG \\ Factores Relacionados con la Demora en el Diagnóstico de Cáncer de Boca y \\ Orofaringe en Juiz de Fora/MG
}

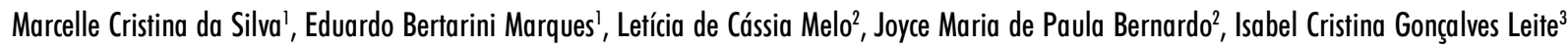

\section{Resumo}

O objetivo do presente estudo foi evidenciar o itinerário que pacientes com câncer de boca e orofaringe percorrem até o diagnóstico e início do tratamento, descrevendo possíveis fatores que podem tornar tardia a identificação dos casos, assim como o início da intervenção. Foi utilizado o modelo de Cuidado à Saúde de Kleinman que inclui os subsistemas: profissional, familiar e popular. Participaram do estudo 37 pacientes e os dados foram obtidos através de entrevista semiestruturada com análise quali-quantitativa dos dados. Em média, os entrevistados conviveram com os sinais/sintomas por 11,4 meses sem diagnóstico. Os pacientes de zona rural, solteiros e do sexo masculino foram os que exibiram maior atraso no diagnóstico. Concluiu-se com o estudo que para se fazer o diagnóstico precoce do câncer oral é preciso intervir nos fatores que influenciam tanto o atraso por parte do paciente quanto do profissional e, assim, destaca-se a necessidade de políticas de saúde, visando ao esclarecimento da população sobre o câncer de boca, e a elaboração de rotinas programadas nos serviços de saúde para a detecção precoce de lesões sugestivas da doença.

Palavras-chave: Saúde Bucal; Evolução Clínica; Neoplasias Bucais; Diagnóstico Precoce

${ }^{1}$ Faculdade de Medicina/ Universidade Federal de Juiz de Fora (MG), Brasil.

${ }^{2}$ Faculdade de Odontologia/Universidade Federal de Juiz de Fora (MG), Brasil.

${ }^{3}$ Professora adjunta Faculdade de Medicina/ Universidade Federal de Juiz de Fora (MG), Brasil. Doutora em Saúde Pública.

Universidade Federal de Juiz de Fora, Núcleo de Assessoria, Treinamento e Estudos em Saúde (NATES) - Campus Universitário.

Endereço para correspondência: Marcelle Cristina da Silva. Rua dos Guararapes, 302/301 - Benfica - Juiz de Fora (MG), Brasil. CEP: 36090-370.

E-mail: marcellecristina_jf@yahoo.com.br 


\section{INTRODUÇÃO}

Anualmente, são diagnosticados cerca de 6,4 milhões de casos de tumores malignos no mundo, sendo o câncer oral responsável por $10 \%$ dos casos ${ }^{1}$. Segundo a Organização Mundial da Saúde (OMS), os cânceres de boca e orofaringe são considerados as neoplasias mais frequentes de cabeça e pescoço, com cerca de 390 mil novos casos por ano ${ }^{1}$. Para o ano de 2008, a incidência estimada para Minas Gerais entre homens é de 8,46 e entre mulheres de 3,07/100.000 habitantes/sexo ${ }^{2}$.

O diagnóstico precoce de câncer bucal e o imediato encaminhamento do paciente para tratamento são fatores importantes para a redução da morbidade e mortalidade causadas pela doença ${ }^{3,4}$. Segundo o estudo de Ries et al..$^{5}$, a chance de sobrevida em cinco anos é de $50 \%$ ou menos, porém, se a lesão for diagnosticada ainda pequena e localizada, as taxas de $70 \%$ a $90 \%$ podem ser atingidas. Contudo, numerosos estudos sugerem que até $50 \%$ dos pacientes apresentam a doença em estágio avançado no momento do diagnóstico. Acredita-se que a natureza silenciosa das lesōes e o atraso no diagnóstico são fatores importantes para esse alto índice de doença avançada ${ }^{6,7,8,9}$.

Existem muitos estudos que revelam atraso do diagnóstico do câncer oral, mas os fatores envolvidos ainda não foram claramente evidenciados. Tais fatores podem estar relacionados tanto com a capacidade do profissional de saúde em detectar precocemente as lesões quanto ao tempo em que o paciente leva para perceber o seu adoecimento e procurar auxílio profissional ${ }^{8}$.

Uma das possibilidades de análise das práticas e estratégias da população no enfrentamento de seus problemas de saúde é o uso dos itinerários terapêuticos, que tem como principal objetivo interpretar os processos pelos quais os indivíduos ou grupos sociais escolhem, avaliam e aderem ou não a determinadas formas de tratamento ${ }^{10,11}$.

Esse processo de escolha pode ser analisado pelo Sistema de Cuidado à Saúde, proposto por Kleinman ${ }^{12}$. Esse Sistema é constituído internamente pela interação de três subsistemas: Subsistema Familiar, Subsistema Profissional e Subsistema Popular, que caracterizam o itinerário terapêutico, no qual as pessoas passam de um subsistema para o outro em diferentes processos de escolha e decisão para o diagnóstico e tratamento da sua doença. O Subsistema Familiar consiste do saber do senso comum, da cultura popular. Inclui neste contexto, o indivíduo e suas redes sociais, como familiares, amigos e vizinhos. Nesse subsistema, são tomadas as primeiras decisóes e cuidados com a doença, como repouso, automedicação, alteração da dieta, remédios caseiros e suporte emocional. O Subsistema Popular refere-se aos especialistas de cura não reconhecidos legalmente e que têm amplo reconhecimento pela sociedade. Geralmente, utilizam recursos como o uso de chás, ervas, cirurgias espirituais, exercícios espirituais e rituais de cura. $\mathrm{O}$ Subsistema Profissional é aquele no qual se localizam os profissionais de cura organizados, legalmente reconhecidos e com aprendizagem formal. $\mathrm{Na}$ maioria das sociedades, a biomedicina é a única representante desse subsistema, porém, em algumas sociedades, existem outros sistemas médicos profissionais como a medicina chinesa tradicional $^{12}$.

Acredita-se que os estudos sobre itinerário terapêutico possam desempenhar um papel importante para compreender como as pessoas constroem seus próprios caminhos no enfrentamento das exigências e consequências do adoecimento e, assim, identificar possíveis erros nesse processo ${ }^{10,11}$. Através deste estudo, buscou-se evidenciar o itinerário que pacientes com câncer de boca e orofaringe, diagnosticados em Juiz de Fora (MG), no período compreendido de janeiro de 2006 a julho de 2007, percorrem até o diagnóstico e início do tratamento, bem como descrever possíveis fatores que podem tornar tardia a identificação dos casos e o início da intervenção.

\section{MÉTODOS}

A análise qualitativa adotada no presente estudo utilizou abordagem teórica compreensiva da Antropologia, dado que a utilização do Sistema de Cuidado à Saúde de Kleinman requer à análise de categorias que são articuladas como experiências culturalmente construídas ${ }^{12}$, portanto, analisa fenômenos não quantificáveis e que não podem ser reduzidos somente à operacionalização matemática de variáveis.

A pesquisa foi submetida ao Comitê de Ética em Pesquisas Humanas da Universidade Federal de Juiz de Fora, em respeito à Resolução 196/96 do Conselho Nacional de Saúde, recebendo aprovação sob parecer $n^{\circ} 166 / 2007$.

Após aprovação das direções das referidas instituiçôes e assinatura do termo de consentimento livre e esclarecido por parte dos casos selecionados, foi realizada a busca ativa a partir de casos em dois hospitais referenciais do município de Juiz de Fora (MG). Ambos os hospitais selecionados são Centros de Alta Complexidade Oncológica (CACON), sendo um do tipo I e o outro do tipo II.

O presente estudo transversal, de base hospitalar, reuniu uma amostra constituída por 37 pacientes, que tiveram o diagnóstico de câncer de boca e orofaringe confirmado no período de janeiro de 2006 a julho de 2007. 
Realizou-se a seleção dos sujeitos da pesquisa a partir da identificação dos casos de interesse no centro de documentação das instituiçôes. Do total de 95 casos identificados nos registros hospitalares, foram selecionados 37 casos que não evoluíram a óbito e que puderam ser contatados para a entrevista.

Os dados foram obtidos através da entrevista semiestruturada, realizada no ambiente do próprio hospital, em espaço reservado para esse fim, com o objetivo de identificar variáveis preditoras clássicas para os cânceres de boca e orofaringe (tabagismo e etilismo), bem como registrar a descrição do itinerário terapêutico de cada caso, desde o início dos sinais e sintomas (tempo de surgimento e tipo de sinal/sintoma), profissionais procurados, medicamentos usados (prescritos ou automedicação) e serviços procurados. As informações foram registradas através da gravação em fita cassete das entrevistas realizadas.

A aplicação do modelo de Cuidado à Saúde de Kleinman ${ }^{12}$ foi realizada após leituras minuciosas das entrevistas, com a finalidade de formação de categorias, dispensando maior atenção ao conjunto de palavras ou expressões com características similares que levaram à compreensão do itinerário terapêutico percorrido pelos pacientes entrevistados até o diagnóstico.

A análise descritiva dos dados quantitativos obtidos foi realizada a partir do programa Epi Info 3.3.2, com nível de significância estatística de 5\%, utilizando o teste $t$ de student $\chi^{2}$ para comparação de médias e proporções entre categorias analisadas.

\section{RESULTADOS}

O perfil demográfico e dos hábitos relacionados ao estilo de vida estão expressos na tabela 1 . Houve predomínio do sexo masculino e a idade média foi de 60 anos. Quanto à declaração da ocupação exercida, revelou-se o predomínio de atividades braçais como "lavrador", "caminhoneiro" e "serviços gerais". Em relação à renda familiar, 54,6\% informaram renda inferior a um salário mínimo.

A amostra exibiu prevalência expressiva de tabagistas e etilistas, sendo que o relato de consumo diário álcool foi feito por $72,7 \%$ dos casos (tabela 1 ).

O uso de prótese dentária foi referido por $54,5 \%$ dos entrevistados.

A tabela 2 apresenta características relativas ao tipo histopatológico e localização anatômica dos tumores. Em 72,7\% dos casos, o diagnóstico foi realizado com o tumor já no estádio IV.

Os sintomas mais frequentes foram dor $(33,3 \%) \mathrm{e}$ disfagia/odinogafia (20\%). Os sinais mais comuns: "caroço no local" (52,9\%), "placa branca" $(29,4 \%)$ e "inchaço" (11,7\%).

Em média, os entrevistados conviveram com os sinais/ sintomas por 11,4 meses sem procurar assistência profissional. Entre as mulheres, a média de tempo foi de 5,5 meses, enquanto, entre os homens, o tempo médio foi de 12,5 meses $(p=0,001)$. Os pacientes solteiros e que declararam morarem sozinhos conviveram com os sintomas por um período mais longo (média de 15 meses).

O primeiro subsistema utilizado foi o Familiar, com a utilização de medicação caseira em $30 \%$ dos casos, como bicarbonato de sódio, violeta e chás ("chá de laranja", "chá de folha de mexerica", "chá de romã",

Tabela 1. Caracterização da amostra, Juiz de Fora, Brasil, 2008

\begin{tabular}{l|c|c}
\hline \multicolumn{3}{c}{ CARACTERIZAÇÃO DA AMOSTRA } \\
\hline \multicolumn{1}{c}{ Variáveis } & Frequência \\
\hline $\begin{array}{l}\text { Sexo } \\
\quad \text { Masculino }\end{array}$ & 32 & $86,5 \%$ \\
Feminino & 5 & $13,5 \%$ \\
\hline $\begin{array}{l}\text { Idade (anos) } \\
\quad<50 \text { anos }\end{array}$ & & \\
$\quad>50$ anos & 8 & $21,6 \%$ \\
\hline $\begin{array}{l}\text { Grau de instrução } \\
\text { Até ensino fundamental } \\
\text { incompleto }\end{array}$ & 29 & $78,4 \%$ \\
\hline $\begin{array}{l}\text { Acima de ensino fundamental } \\
\text { completo }\end{array}$ & 24 & $64,8 \%$ \\
\hline $\begin{array}{l}\text { Situação profissional } \\
\text { Ativo } \\
\text { Aposentado (idade ou invalidez) }\end{array}$ & 13 & $35,2 \%$ \\
\hline $\begin{array}{l}\text { Tabagismo } \\
\text { Presente } \\
\text { Ausente }\end{array}$ & 11 & $29,7 \%$ \\
\hline Etilismo & 31 & $83,8 \%$ \\
$\quad \begin{array}{l}\text { Sim } \\
\text { Não }\end{array}$ & 6 & $16,2 \%$ \\
\hline
\end{tabular}

Tabela 2. Características tumorais, Juiz de Fora, Brasil, 2008

\begin{tabular}{l|c|c}
\hline \multicolumn{3}{c}{ CARACTERÍSTICAS TUMORAIS } \\
\hline \multicolumn{2}{c}{ Variáveis } & Frequências \\
\hline Tipo histopatológico & 36 & \\
Carcinoma de células escamosas & 36 & $97,3 \%$ \\
Outros tipos & 1 & $2,7 \%$ \\
\hline Localização tumoral & & \\
Língua & 15 & $40,5 \%$ \\
Assoalho de boca & 8 & $21,6 \%$ \\
Orofaringe & 6 & $16,2 \%$ \\
Outros & 8 & $21,7 \%$ \\
\hline
\end{tabular}


"chá de transage", "chá de folha de algodão"). A frequência de utilização de chás foi maior nos pacientes que residem em zona rural $(\mathrm{p}=0,001)$. O tempo médio, desde o início dos sintomas até a decisão por busca da ajuda profissional, diferiu significativamente entre pacientes oriundos das áreas rurais e das áreas urbanas $(\mathrm{p}=0,001)$, sendo que as médias respectivas foram $14 \mathrm{e}$ 8,8 meses.

A utilização do Subsistema Popular foi relatada por $8,1 \%$ dos entrevistados e a procura foi por curandeiros e cirurgias espirituais.

Em relação ao Subsistema Profissional, o médico foi o primeiro profissional a ser procurado em $64,3 \%$ dos casos, seguido pelo dentista $(28,6 \%)$ e farmacêutico $(7,1 \%)$. Em 45,5\% dos casos, houve encaminhamento para outro profissional médico/dentista e, em $11 \%$, exclusivamente tratamento sintomático, com a utilização medicaçôes como analgésicos e anti-inflamatórios. Após a procura pelo primeiro profissional, o tempo médio para a realização da biópsia e o diagnóstico foi de dois meses. O tempo médio entre o diagnóstico histopatológico e início do tratamento foi de 45 dias.

\section{DISCUSSÃO}

O perfil da amostra estudada condiz com a literatura vigente $^{3,5,6,7,8}$, caracterizada pelo predomínio de homens, acima de 50 anos, com baixa escolaridade e com tumores em diagnóstico avançado. $\mathrm{Na}$ investigação dos fatores envolvidos com o atraso no diagnóstico de câncer oral, deve-se estar atento a todos os caminhos percorridos pelo paciente. Esse processo consiste na percepção dos primeiros sinais e sintomas, na busca por atendimento inicial, no reconhecimento pelo profissional de saúde do adoecimento, na realização da biópsia, no encaminhamento para o especialista e, finalmente, no diagnóstico definitivo de câncer e implementação do tratamento indicado. Assim, o reconhecimento tardio das lesóes pode estar associado ao atraso do paciente e ao atraso do profissional de saúde; e os fatores envolvidos podem ser organizados e analisados segundo a lógica dos subsistemas de saúde.

$O$ primeiro passo na procura por atendimento é a percepção dos sinais e sintomas pelo paciente. A partir desse reconhecimento, aspectos relacionados aos Subsistemas Familiar, Popular e Profissional influenciarão na tomada de decisóes pelo paciente e no caminho percorrido por ele até o diagnóstico.

De acordo com Adlard e Hume ${ }^{13}$, um dos fatores que contribuem para o atraso é a falta do conhecimento dos principais sinais e sintomas do câncer oral por parte dos pacientes. Kowalski ${ }^{14}$ constatou em seu estudo que
66\% dos pacientes somente procuraram assistência médica devido à persistência de sintomas inexplicáveis ou quando tais sintomas causavam incômodo excessivo. Esses fatos podem ser observados nos depoimentos transcritos literalmente:

Eu uso dentadura e comecei a notar um caroço embaixo dela. No começo não me preocupei muito e depois de uns quatro meses começou a inchar e aí sim fiquei preocupado e fui no dentista (masculino, 63 anos).

Apareceu um caroço na minha boca. Esse caroço não doía e nem saía nada nele. Foi por isso que não procurei o médico, achei que era uma pereba dessas aí, afta, sei lá. Mas não melhorou e o caroço continuou crescendo e começou a doer. Minha mulher ficou preocupada e me mandou ir no médico (masculino, 81 anos).

As manifestações iniciais do tumor também influenciam na percepção do paciente quanto ao seu adoecimento. No estudo de Onizawa et al. ${ }^{8}$, pacientes com úlceras ou lesões brancas mostraram maior atraso na procura por assistência do que aqueles com dor ou inchaço. No estudo de Carvalho et al..$^{15}$, o sexo feminino foi associado a um risco menor de ser diagnosticado com a doença em estágio avançado, enquanto que os doentes solteiros apresentaram um risco superior. Esses achados corroboram os dados do presente estudo. Isso pode ser explicado pelo estudo de Celile et al. ${ }^{16}$, que analisa as diferenças entre homens e mulheres no cuidado à saúde. Segundo os autores, pacientes do sexo feminino apresentam maior sensibilidade na percepção dos sinais e sintomas, que, aliada a um maior cuidado corporal, faz com que esse grupo busque assistência mais antecipadamente.

Estimativas indicam que aproximadamente $30 \%$ dos pacientes demoram mais que três meses após a autodescoberta dos sintomas do câncer oral para procurar ajuda profissional ${ }^{9,8,17,18}$. Esse fato é corroborado pelos achados do presente estudo, que apresentou uma média de 11,4 meses. No contexto do Subsistema Familiar, os aspectos identificados como fatores que influenciaram o paciente a procurar assistência de saúde foram principalmente o estado civil, o uso de chás e o local de moradia (rural ou urbana).

No estudo de Grunfeld et al. ${ }^{6}$, não houve associação entre o status marital e o atraso no diagnóstico por câncer. Contudo, os resultados aqui apontados indicam o mesmo efeito descrito por outros autores ${ }^{19,20}$, que exibem resultados que associam indivíduos solteiros com menor busca por atendimento odontológico, além de terem maior probabilidade de usarem tabaco, álcool e 
serem expostos a uma dieta pobre. Os pacientes que residem em área rural apresentaram um tempo médio entre o aparecimento dos primeiros sintomas e a procura por ajuda profissional maior que o dos pacientes de área urbana. Isso pode estar relacionado ao baixo nível de informação que esses pacientes apresentam sobre os sinais e sintomas de um câncer oral. Gomes e Carvalho ${ }^{21}$, por meio de uma entrevista domiciliar, avaliaram o nível de informação sobre prevenção do câncer em moradores de regiōes rurais. Os resultados do estudo demonstraram que essa população apresentava um baixo nível de conhecimento sobre a associação entre o câncer e os fatores de risco. Outro possível fator contribuinte devese ao maior consumo de chás feitos por esses pacientes. Segundo o estudo Kerpondon ${ }^{18}$, o uso de chás ou ervas é um fator de atraso significativo no diagnóstico de câncer de boca, por tornar a procura por atendimento mais demorada.

No estudo de Kerdpon ${ }^{18}$, apenas $17 \%$ dos casos analisados buscaram como primeiro atendimento profissional o dentista, e as razóes pela procura foram problemas relacionados aos dentes ou gengivas. No presente estudo, a busca pelo dentista também foi menor, indicada em $28,6 \%$ dos casos. Segundo o autor, isso reflete o entendimento da comunidade de que os dentistas são responsáveis apenas pelos dentes e gengiva. Esse fato pode ser demonstrado por um trecho de uma das entrevistas realizadas:

Comecei a sentir um corte na língua que não me incomodava. Depois de um tempo começou a doer e a me incomodar. Procurei a dentista do posto para ela arrancar o dente que tava encostando na minha língua e machucando e aí ele viu que era um câncer (masculino, 57 anos).

Ainda segundo Kerpdon ${ }^{18}$, não houve diferença no tempo de atraso entre diagnóstico e conduta terapêutica, entre médicos e dentistas, sendo em geral de 51,2 dias, enquanto que no estudo de $S$ chnetler ${ }^{22}$ há o relato que os médicos têm tendência a fazer o diagnóstico mais precoce. Por outro lado, outros relatos indicam que os dentistas identificam doentes numa fase mais inicial ${ }^{23}$.

Segundo Brouha $^{24}$, o atraso profissional varia de quatro dias a 3,5 meses, dependendo da localização e tamanho do tumor. Pacientes com tumor T3 e T4 tiveram, neste estudo, menos atraso profissional no diagnóstico que os pacientes com tumor T1 e T2. Isso demonstra a maior dificuldade dos profissionais em reconhecer lesões cancerosas em seus estágios iniciais. $\mathrm{O}$ atraso profissional no presente estudo foi de dois meses e, em $72,7 \%$ dos casos, o diagnóstico foi realizado com o tumor já no estádio IV, estadiamento feito através do TNM da União Internacional Contra o Câncer (UICC) ${ }^{25}$.

\section{CONCLUSÃO}

Para se fazer o diagnóstico precoce do câncer oral, é preciso intervir nos fatores que influenciam tanto o atraso do paciente quanto o atraso profissional. A demora no reconhecimento dos sinais e sintomas da doença está entre os principais responsáveis pelo atraso do paciente e nessa etapa são necessárias políticas de saúde visando ao esclarecimento da população acerca dos fatores de risco, sinais e sintomas comuns da doença e na importância do autoexame bucal. Como verificado no presente estudo, os pacientes de zona rural e do sexo masculino são os que mais demonstraram atraso no diagnóstico e assim torna-se importante o direcionamento de tais políticas de saúde para esses subgrupos populacionais. $\mathrm{O}$ atraso do profissional está relacionado à falha no reconhecimento dos sinais e sintomas sugestivos de câncer. Sendo assim, é necessária a reflexão dos profissionais diretamente envolvidos nessa problemática sobre a necessidade oportunidades diagnósticas e de educação para saúde com a elaboração de rotinas programadas nos serviços de saúde para a detecção de lesões sugestivas de câncer de boca. É também oportuna a reflexão sobre o desenvolvimento de programas de ensino sobre Oncologia nos cursos de graduação, em especial, na Medicina e Odontologia.

\section{Declaração de Conflito de Interesses: Nada a Declarar}

\section{REFERÊNCIAS}

1. Stewart BW, Kleihues P, editors. World cancer report. Lyon: IARC Press; 2003.

2. Instituto Nacional de Câncer. Estimativa da incidência e mortalidade por câncer no Brasil. 2008. [acesso em 2008 jun 01]. Disponível em http://www.inca.gov.br/

3. Brad WN, Terry AD. Oral Cancer and Precancerous Lesions. CA Cancer J Clin 2002;52: 195- 215.

4. Syme SE, Drury TF, Horowitz AM. Maryland dental hygienists knowledge and opinions of cancer risk factors and diagnostic procedures. Oral Dis 2001;7:177-84.

5. Ries L, Eisner M, Kosary C, Hankey B, Miller B, Clegg L, et al. SEER cancer statistics review, 1973-1997. Bethesda, MD: National Cancer Institute.2000.

6. Scott SE, Grunfeld EA, McGurk M. The idiosyncratic relationship between diagnostic delay and stage of oral squamous cell carcinoma. Oral Oncol 2005;41:396-403.

7. Wildt J, Bundgaard T, Bentzen SM. Delay in the diagnosis of oral squamous cell carcinoma. Clin Otolaryngol 1995; 20 (1): $21-5$. 
8. Onizawa K, Nishihara K, Yamagata K, Yusa H, Yanagawa T, Yoshida $\mathrm{H}$. Factors associated with diagnostic delay of oral squamous cell carcinoma. Oral Oncol 2003;39:781-8.

9. Scott SE, Grunfeld EA, McGurk M. Patient's delay in oral cancer: a systematic review. Community Dent Oral Epidemiol 2006; 34: 337-43.

10. Gerhardt TE. Itinerários terapêuticos em situações de pobreza: diversidade e pluralidade. Cad Saude Publica 2006; 22(11): 2449-63.

11. Duarte LFD, Leal OF. Doença, sofrimento, perturbação, perspectivas etnográficas. Rio de Janeiro: FIOCRUZ; 1998. 210p.

12. Kleinman A. Concepts and a model for the comparision of medical systems as cultural systems. Soc Sci Med 1978; 12: 85-73.

13. Adlard JW, Hume MJ. Cancer knowledge of the general public in the United Kingdom: survey in a primary care setting and review of the literature. Clin Oncol. 2003; 15:174-80.

14. Kowalski LP, Franco EL, Torloni H, Fava AS, Andrade Sobrinho J, Ramos G, et al. Lateness of diagnosis of oral and oropharyngeal carcinoma: factors related to the tumour, the patient and health professionals. Eur J Cancer B Oral Oncol 1994; 30B:167-73.

15. Carvalho AL, Schlecht NF, Fava AS, Franco EL, et al. Predictive factors for diagnosis of advanced-stage squamous cell carcinoma of the head and neck. Arch Otolaryngol Head and Neck Surg 2002; 128: 313-8.
16. Van Wijk G, Kolk AM. Sex differences in physical symptoms: the theory contribution of symptom perception theory. Soc Sci Med 1997; 45:231-46.

17. Hollows P, McAndrew PG, Perini MG. Delays in the referral and treatment of oral squamous cell carcinoma. Br Dent J 2000;188:262-5.

18. Kerdpon D, Sriplung H. Factors related to delay in diagnosis of oral squamous cell carcinoma in southern Thailand. Oral Oncol 2001;37:127-31.

19. Manski RJ. Dental care coverage among older americans. J Am Coll Dent 1995; 62: 41-4

20. Osterberg T, Lundgren M, Emilson CG, Sundh V, Birkhed D, Steen B. Utilization of dental services in relation to socioeconomic and health factors in middle aged and elderly in Swedish population. Acta Odontol Scand 1998; 56: 41-7.

21. Gomes UA, Carvalho EM. O conhecimento da população sobre prevenção do cancer no Brasil . Revista brasileira de cancerologia 1999; 45(3):29-37.

22. Schnetler JF. Oral cancer diagnosis and delays in referral. $\mathrm{Br}$ J Oral Maxillofac Surg 1992; 30(4):210-3.

23. Referral delay in diagnosis of oro/oro-pharyngeal cancer in Israel. Eur J Cancer B Oral Oncol 1995;31B(3):166-8.

24. Brouha XD, Tromp DM, Koole R, Hordijk GJ, Winnubst JA, Leeuw JR. Profissional delay in head and neck cancer patients: Analysis of the diagnosis pathway. Oral Oncology 2007; 43: 551-6.

25. União Internacional Contra o Câncer. TNM: classificação de tumores malignos. Rio de Janeiro: UICC; 1997. 


\section{Abstract}

The present study aimed to evidence the route that patients with oral and oropharynx cancer take before diagnosis and beginning of the treatment, describing possible factors that can lead to a late identification of cases, as well as the beginning of its intervention. The Kleinman's health care model was used, which includes professional, family and popular sub-systems. 37 patients participated in the study and the data was obtained through a semi-structured interview with a quail-quantitative analysis of data. On average, the interviewees lived with the signs/symptoms for 11,4 months without diagnosis. Single and male patients from rural areas were those who demonstrated the longest delay for the diagnosis. The study concludes that, in order to early diagnose oral cancer it is necessary to interfere with factors that influence both professional and patients delay. Thus it is necessary to implement health care policies with the objective of educating the population about oral cancer and design programmed routines in health care services for the early detection of suggestive lesions of this disease.

Key words: Oral Health; Clinical Evolution; Mouth Neoplasms; Early Diagnosis

\section{Resumen}

El objetivo de este estudio fue resaltar la ruta que siguen los pacientes con cáncer de boca y orofaringe hasta el diagnóstico y el comienzo del tratamiento, describiendo los posibles factores que pueden retardar la identificación de los casos, así como el comienzo de la intervención. Se utilizó el modelo de Sistema de Cuidado a la Salud de Kleinman, que incluye los subsistemas: profesional, familiar y popular. Participaron del estudio 37 pacientes y los datos fueron obtenidos a través de entrevista semiestructurada con un análisis cualitativo y cuantitativo de los datos. En promedio, los encuestados convivieron con las señales o síntomas durante 11,4 meses sin diagnóstico. Los pacientes de las zonas rurales, solteros y del sexo masculino mostraron una mayor demora en el diagnóstico. Se concluye con el estudio, que para llevar a cabo el diagnóstico precoz del cáncer oral se necesita intervenir en los factores que influyen tanto en el retraso por parte del paciente, como en el del profesional, poniendo de manifiesto la necesidad de políticas de salud dirigidas a aclarar la población sobre el cáncer de boca, y el desarrollo de rutinas programadas en los servicios de salud para la detección temprana de lesiones sugestivas de la enfermedad.

Palabras clave: Salud Bucal; Evolución Clínica; Neoplasias de la Boca; Diagnóstico Precoz 